\title{
The Prognostic Value of Postoperative Lymph Node Ratio in Gastric Adenocarcinoma Patients Treated With Neoadjuvant Chemotherapy
}

Abdullah Sakin ${ }^{1}$, Muhammed M. Atci ${ }^{1}$, Mehmet Naci Aldemir ${ }^{2}$, Baran Akagündüz ${ }^{3}$, Suleyman Şahin ${ }^{4}$, Serdar Arıc1 ${ }^{5}$, Saban Secmeler ${ }^{6}$, Sener Cihan ${ }^{1}$

1. Medical Oncology, Prof. Dr. Cemil Taşcıoğlu City Hospital, Istanbul, TUR 2. Medical Oncology, Yüzüncü Yıl University, Van, TUR 3. Medical Oncology, Erzincan Binali Yıldırım Üniversitesi Mengücek Gazi Hastanesi, Erzincan, TUR 4. Medical Oncology, Van Research and Training Hospital, Van, TUR 5. Medical Oncology, Şișli Etfal Research Hospital, Istanbul, TUR 6. Medical Oncology, Şanlıurfa Research Hospital, Şanlıurfa, TUR

Corresponding author: Abdullah Sakin, drsakin@hotmail.com

\section{Abstract}

\section{Objective}

In this study, we aimed to investigate the prognostic value of postoperative lymph node ratio (LNR) in locally advanced gastric cancer (GC) patients receiving neoadjuvant chemotherapy (NACT).

\section{Methods}

LNR was calculated as the ratio of positive LNs to the total LNs removed. The receiver operating characteristic (ROC) curve was plotted to estimate the cut-off value of LNR for recurrence. The area under the curve of LNR was 0.714 (95\% CI: $0.604-0.825$, p<0.001) with $60 \%$ sensitivity and $>0.255$ with $76 \%$ specificity. Patients were grouped as group I $(\leqslant 0.255)$ and group II $(>0.255)$.

\section{Results}

In this study, $157 \mathrm{GC}$ patients were included (39.5\% female and 60.5\% male). Of the patients, 97 (61.8\%) were in group I and 60 (38.2\%) were in group II. Disease-free survival (DFS) was not reached in group I, and it was 16 months in group II ( $\mathrm{p}<0.001)$. Overall survival (OS) was 58 months in group I and 28 months in group II $(\mathrm{p}>0.001)$. In multivariate analysis, lymphovascular invasion, neoadjuvant response, adjuvant treatment, and LNR were found to be the factors associated with DFS and OS ( $<<0.05)$.

\section{Conclusion}

In our study, it was observed that LNR can predict survival rates better than LN staging.

Review began 03/23/2021 Review ended 04/13/2021 Published 04/22/2021

\section{() Copyright 2021}

Sakin et al. This is an open access article distributed under the terms of the Creative Commons Attribution License CC-BY 4.0., which permits unrestricted use, distribution, and reproduction in any medium, provided the original author and source are credited.
Categories: Oncology

Keywords: gastric cancer, neoadjuvdnt, staging, lymph node ratio, prognostic

\section{Introduction}

The incidence of gastric cancer (GC) has been decreasing since the 1930s; however, it still remains a major cause of cancer-related deaths globally. Most GC patients are symptomatic and have a locoregional or advanced-stage disease at the time of diagnosis, but despite the advances in treatment modalities, only half of those patients with locoregional tumors are able to undergo potentially curative resection [1-3].

Prospective randomized trials and meta-analyses have indicated improved survival with multimodality approaches, such as adjuvant chemoradiotherapy, adjuvant chemotherapy (ACT), and neoadjuvant chemotherapy (NACT) compared to surgery alone. The positive effects of these therapies on survival outcomes in GC patients have become prominent over time, although there is no consensus as to which is the best approach [4-8].

There are two major staging systems related to GC, which are as follows: (i) the Japanese classification based on anatomical location, especially of the lymph node (LN) stations, and (ii) the tumor, node, and metastasis (TNM) staging system developed jointly by the American Joint Committee on Cancer (AJCC) and the Union for International Cancer Control (UICC). The recent AJCC/UICC TNM staging classification (eighth edition, 2017 ) includes another prognostic stage group for clinical and pathological staging following NACT $[9,10]$.

In the TNM classification system, LN staging is based on the total number of metastatic LNs and does not take into account the number of total LNs removed. In addition, it may cause a limitation in both tumor staging and prediction of survival, especially in patients with inadequate LN dissection [11]. 
In Japan, D2 dissection has been recommended as standard practice since the 1960s. East Asian surgeons, especially Japanese and Korean surgeons, have routinely performed gastrectomy with D2 dissection. However, most Western surgeons perform gastrectomy with only D1 dissection, because D1 was reportedly associated with less mortality and morbidity than D2 in prospective randomized trials performed in the Netherlands and the UK, which concluded that there was no survival benefit for D2 over D1 LN dissection $[12,13]$.

The debate on lymphadenectomy and the extent of the total number of LNs removed began in the 1980s. The National Comprehensive Cancer Network (NCCN) guidelines recommend the examination of 16 or more regional LNs to determine the N status. Several studies have demonstrated a robust association between the number of LNs removed and improved survival rates [14,15].

LN metastasis was the only independent predictor of survival in patients treated with NACT in the Medical Research Council Adjuvant Gastric Infusional Chemotherapy (MAGIC) trial, and pathological response to chemotherapy was not associated with survival [16]. The LN ratio (LNR) is calculated as the ratio of positive LNs to the total LNs removed. Previous studies and meta-analyses have confirmed that LNR is an independent prognostic factor for overall survival (OS) in GC patients who undergo surgery without NACT [17-20]. In the current study, we aimed to investigate the prognostic value of postoperative LN ratio (ypLNR) in locally advanced GC patients receiving NACT.

\section{Materials And Methods Study population}

The patients who were followed up at the Yüzüncü Yıl University Faculty of Medicine and Prof. Dr. Cemil Taşcıoğlu Istanbul City Training and Research Hospital between 2010 and 2019 were included in the study. Patients who met any of the following criteria were excluded: metastatic stage, unoperated patients, patients with disease progression during NACT, those with complete response to NACT, surgical margin-positive patients, those who were $<18$ years of age, patients operated on without NACT, patients who were LN-negative after NACT, those with a history of a second primary cancer, and those with missing data. A total of 653 patient files were reviewed, and 157 patients were ultimately recruited for the study (Figure 1). Patients were restaged according to the AJCC Cancer Staging Manual, 8th edition.

\section{Data collection}

Demographics, clinicopathological features, laboratory parameters, and treatment-related data of the patients were obtained from documented archive files. Gastric tumor localization was categorized into four groups as (1) upper 1/3 (gastroesophageal junction and cardia), (2) middle 2/3 (corpus), (3) lower 3/3 (antrum and pyloric), and (4) linitis plastica. NACT was grouped as FLOT4 (docetaxel, oxaliplatin, and fluorouracil/leucovorin) and others [ECF (epirubicin, cisplatin, and fluorouracil/leucovorin), ECX (epirubicin, cisplatin, capecitabine), EOF (epirubicin, oxaliplatin, and fluorouracil/leucovorin), and EOX (epirubicin, oxaliplatin, capecitabine)]. Tumor regression was assessed as near-complete (fibrosis and rare residual tumor cells in the specimen), partial response (fibrosis outgrowing residual tumor in the specimen), poor response (rare fibrosis and residual tumor outgrowing fibrosis and tumor without evidence of regressive changes). LN ratio (ypLNR) was calculated as the ratio of positive LNs to the total LNs removed.

\section{Ethics committee approval}

This study was conducted in accordance with the Declaration of Helsinki and reviewed and approved by the Ethics Committee of the University of Health Sciences, Prof. Dr. Cemil Taşcıoglu Istanbul City Training and Research Hospital (46870771-514.10-12.10.2020).

\section{Statistical analysis}

SPSS Statistics 22.0 for Windows software (IBM Corp., Armonk, NY) was used for the statistical analysis. Descriptive analyses were described as mean, standard deviation, and minimum and maximum values for numerical variables while categorical variables were presented as numbers and percentages. The student's t-test was used when numerical variables had normal distribution in two independent groups, and the Mann-Whitney U test was used in the absence of normal distribution. Chi-square analysis was used to compare the ratios in the groups. Monte Carlo simulation was applied when the conditions were not met. Survival analyses were performed by the Kaplan-Meier method. The determinant factors were examined by Cox regression analysis. The ENTER model was used for the factors with $\mathrm{p}<0.05$ as determined in the univariate analysis. The receiver operating characteristic (ROC) curve was plotted to estimate the optimal cut-off value of ypLNR for recurrence. Area under the curve of ypLNR was 0.714 (95\% CI: 0.604-0.825, $\mathrm{p}<0.001$ ) with $60 \%$ sensitivity and $>0.255$ with $76 \%$ specificity (Figure 2 ). Patients were grouped according to ypLNR as group 1 (ypLNR of $\leqslant 0.255$ ) and group 2 (ypLNR of $>0.255$ ). Disease-free survival (DFS) was calculated as the time from the initiation of treatment to progression. Overall survival (OS) was calculated as the time from the date of diagnosis to the date of death or last follow-up. Cut-off values were determined according to the ROC curve analysis. An overall 5\% alpha error level was used to infer statistical significance. A p-value of $<0.05$ was considered statistically significant. 


\section{Results}

The study population consisted of 157 eligible patients; 62 (39.5\%) of them were female and 95 (60.5\%) were male. The patients' median age was 58 years (range: 26-75 years). The clinical stage was 3 in 151 patients (96.2\%). According to the Lauren classification, 129 patients (83.2\%) were classified as intestinal-type, and gastric localization was upper in 77 patients (49.0\%). The histological type was tubular and papillary adenocarcinoma in 100 patients (63.7\%). Seventy-five patients (47.8\%) had undergone total gastrectomy, and 78 (49.7\%) had undergone D2 LN dissection. The ypTNM stage was I in 10 patients (6.4\%), II in 42 (26.8\%), and III in 105 patients (66.9\%). A total of 144 patients (91.7\%) had been able to complete NACT. During the follow-up, 60 patients had recurrence (38.2\%) and 46 patients died (29.3\%). Clinical and pathological data of the patients and comparison of data by LNR groups are summarized in Table 1 .

According to the Kaplan-Meier curve, median DFS was not reached in group 1, and in group 2, it was 16 months (95\% CI: 12.9-20.5) (log-rank p<0.001) (Figure 3). Median OS was 58 months in group 1 and 28 months in group 2 (95\% CI: 12.9-43.0) (log-rank p<0.001) (Figure 4).

According to the univariate analysis, the factors that affected DFS were carcinoembryonic antigen (CEA) $(p=0.044)$, ring cell carcinoma histology $(p=0.010)$, D2 LN dissection $(p=0.032)$, perineural invasion $(\mathrm{p}=0.005)$, lymphovascular invasion $(\mathrm{p}=0.002), \operatorname{ypTNM}(\mathrm{p}=0.001), y \mathrm{pT}(\mathrm{p}=0.033), \mathrm{yp}(\mathrm{p}<0.001)$, number of involved LNs $(p<0.001)$, NACT response $(p<0.001)$, NACT completion $(p=0.006)$, and LNR $(p<0.001)$; while the factors that affected OS were found to be distal tumor localization and linitis plastica $(\mathrm{p}=0.024$ and $\mathrm{p}=0.022$, respectively), signet ring cell histology $(\mathrm{p}=0.010)$, perineural and lymphovascular invasion $(\mathrm{p}=0.013$ and $\mathrm{p}=0.007$, respectively), ypN ( $\mathrm{p}=0.004)$, the number of positive LNs $(\mathrm{p}<0.001)$, neoadjuvant treatment response $(\mathrm{p}<0.001)$, NACT completion $(\mathrm{p}=0.001)$, and ypLNR $(\mathrm{p}=0.001)$ (Table 2$)$.

Among the factors found to affect DFS in the univariate analysis, CEA, histology, LN dissection method, perineural invasion, lymphovascular invasion, ypT, ypN, the number of involved LNs ( $<<0.001)$, NACT response, NACT completion, and ypLNR were used to create a model, which showed lymphovascular invasion $(p=0.002)$, NACT response $(p<0.001)$, NACT completion $(p=0.006)$, and ypLNR $(p<0.001)$ as the associated factors. Similarly, among the factors found to affect OS in the univariate analysis, tumor localization, histology, perineural and lymphovascular invasion, ypN, neoadjuvant treatment response, NACT completion, and ypLNR ( $\mathrm{p}=0.001$ ) were used for a multivariate analysis, which showed linitis plastica $(\mathrm{p}=0.022)$, neoadjuvant treatment response $(\mathrm{p}<0.001)$, NACT completion $(\mathrm{p}=0.001)$, and ypLNR $(\mathrm{p}=0.001)$ as the factors that affected the OS (Table 3).

\begin{tabular}{|c|c|c|c|c|c|c|c|c|}
\hline \multirow{2}{*}{ Variables } & & \multicolumn{2}{|c|}{$\begin{array}{l}\text { All patients } \\
(n=157)\end{array}$} & \multicolumn{2}{|c|}{$\begin{array}{l}\text { Group I } \\
\text { (n=97) }\end{array}$} & \multicolumn{2}{|c|}{$\begin{array}{l}\text { Group II } \\
(n=60)\end{array}$} & \multirow{2}{*}{$\begin{array}{l}\mathrm{P} \text { - } \\
\text { value }\end{array}$} \\
\hline & & $\mathrm{N}$ & $\%$ & $\mathbf{N}$ & $\%$ & $\mathrm{~N}$ & $\%$ & \\
\hline \multirow{2}{*}{ Gender } & Women & 62 & 39.5 & 45 & 46.4 & 17 & 28.3 & \multirow{2}{*}{0.024} \\
\hline & Men & 95 & 60.5 & 52 & 53.6 & 43 & 71.7 & \\
\hline Age, years & Median (min-max) & \multicolumn{2}{|c|}{$58(26-75)$} & \multicolumn{2}{|c|}{$60(26-73)$} & \multicolumn{2}{|c|}{57 (33-75) } & 0.629 \\
\hline \multirow{2}{*}{ Smoking status } & Yes & 32 & 20.4 & 15 & 15.5 & 17 & 28.3 & 0.052 \\
\hline & Pack/year, mean \pm SD & \multicolumn{2}{|c|}{$29.5 \pm 14.7$} & \multicolumn{2}{|c|}{$31.1 \pm 16.0$} & \multicolumn{2}{|c|}{$28.0 \pm 13.8$} & 0.655 \\
\hline \multirow{2}{*}{ ECOG PS } & 0 & 141 & 89.8 & 88 & 90.7 & 53 & 88.3 & \multirow{2}{*}{0.631} \\
\hline & 1 & 16 & 10.2 & 9 & 9.3 & 7 & 11.7 & \\
\hline \multirow{2}{*}{ Clinical stage } & 2 & 6 & 3.8 & 4 & 4.1 & 2 & 3.3 & \multirow{2}{*}{0.802} \\
\hline & 3 & 151 & 96.2 & 93 & 95.9 & 58 & 96.7 & \\
\hline CEA & $\mathrm{ng} / \mathrm{mL}$, mean $\pm \mathrm{SD}$ & \multicolumn{2}{|c|}{$10.3 \pm 19.5$} & \multicolumn{2}{|c|}{$9.0 \pm 17.4$} & \multicolumn{2}{|c|}{$12.6 \pm 22.7$} & 0.601 \\
\hline CA 19-9 & $\mathrm{U} / \mathrm{mL}$, mean $\pm \mathrm{SD}$ & \multicolumn{2}{|c|}{$125 \pm 155.9$} & \multicolumn{2}{|c|}{$147.4 \pm 151.5$} & \multicolumn{2}{|c|}{$129.2 \pm 82.5$} & 0.694 \\
\hline \multirow{2}{*}{ Lauren classification } & Intestinal & 129 & 83.2 & 82 & 86.3 & 47 & 78.3 & \multirow{2}{*}{0.195} \\
\hline & Diffuse & 26 & 16.8 & 13 & 13.7 & 13 & 21.7 & \\
\hline \multirow{3}{*}{ Tumor localization } & $1 / 3$ & 77 & 49.0 & 55 & 56.7 & 22 & 36.7 & \multirow{3}{*}{0.007} \\
\hline & $2 / 3$ & 34 & 21.7 & 16 & 16.5 & 18 & 30.0 & \\
\hline & $3 / 3$ & 37 & 23.6 & 24 & 24.7 & 13 & 21.7 & \\
\hline
\end{tabular}




\begin{tabular}{|c|c|c|c|c|c|c|c|c|}
\hline & Linitis plastica & 9 & 5.7 & 2 & 2.1 & 7 & 11.7 & \\
\hline \multirow{3}{*}{ Histology } & $\begin{array}{l}\text { Tubular and papillary } \\
\text { adenocarcinoma }\end{array}$ & 100 & 63.7 & 71 & 73.2 & 29 & 48.3 & \multirow{3}{*}{0.001} \\
\hline & Mucinous carcinoma & 17 & 10.8 & 11 & 11.3 & 6 & 10.0 & \\
\hline & Signet ring cell carcinoma & 40 & 25.5 & 15 & 15.5 & 25 & 41.7 & \\
\hline \multirow{3}{*}{ Grade } & 1 & 6 & 3.8 & 5 & 5.2 & 1 & 1.7 & \multirow{3}{*}{$<0.001$} \\
\hline & 2 & 79 & 50.3 & 60 & 61.9 & 19 & 31.7 & \\
\hline & 3 & 72 & 45.9 & 32 & 33.0 & 40 & 66.7 & \\
\hline \multirow{2}{*}{ Gastrectomy } & Subtotal & 82 & 52.2 & 55 & 56.7 & 27 & 45.0 & \multirow{2}{*}{0.154} \\
\hline & Total & 75 & 47.8 & 42 & 43.3 & 33 & 55.0 & \\
\hline \multirow{2}{*}{ LN dissection } & D1 & 79 & 50.3 & 46 & 47.4 & 33 & 55.0 & \multirow{2}{*}{0.356} \\
\hline & D2 & 78 & 49.7 & 51 & 52.6 & 27 & 45.0 & \\
\hline \multirow{3}{*}{ урTNM } & I & 10 & 6.4 & 10 & 10.3 & 0 & 0.0 & \multirow{3}{*}{$<0.001$} \\
\hline & II & 42 & 26.8 & 37 & 38.1 & 5 & 8.3 & \\
\hline & III & 105 & 66.9 & 50 & 51.5 & 55 & 91.7 & \\
\hline \multirow{2}{*}{ урт } & 1 & 27 & 17.2 & 22 & 22.7 & 5 & 8.3 & \multirow{2}{*}{0.021} \\
\hline & 2 & 130 & 82.8 & 75 & 77.3 & 55 & 91.7 & \\
\hline Tumor diameter & $\mathrm{mm}$, mean $\pm \mathrm{SD}$ & 46.7 & & 37.7 & \pm 20.5 & 60.8 & \pm 38.1 & 0.005 \\
\hline \multirow{3}{*}{ ypN } & 1 & 55 & 35.0 & 54 & 55.7 & 1 & 1.7 & \multirow{3}{*}{$<0.001$} \\
\hline & 2 & 38 & 24.2 & 33 & 34.0 & 5 & 8.3 & \\
\hline & 3 & 64 & 40.8 & 10 & 10.3 & 54 & 90.0 & \\
\hline Number of LNs removed & Median (min-max) & \multicolumn{2}{|c|}{$27(12-78)$} & \multicolumn{2}{|c|}{$28(12-78)$} & \multicolumn{2}{|c|}{$25(13-64)$} & 0.364 \\
\hline \multirow[t]{2}{*}{ Number of positive LNs } & Median (min-max) & \multicolumn{2}{|c|}{$4(1-30)$} & \multicolumn{2}{|c|}{$2(1-11)$} & \multicolumn{2}{|c|}{$12(6-30)$} & 0.001 \\
\hline & $0-1$ & 137 & 87.3 & 83 & 85.6 & 54 & 90.0 & \multirow{3}{*}{0.410} \\
\hline \multirow[t]{2}{*}{ ERBB2 } & 2 & 13 & 8.3 & 8 & 8.2 & 5 & 8.3 & \\
\hline & 3 & 7 & 4.5 & 6 & 6.2 & 1 & 1.7 & \\
\hline Perineural invasion & Positive & 110 & 70.1 & 61 & 62.9 & 49 & 81.7 & 0.013 \\
\hline Lymphovascular invasion & Positive & 111 & 70.7 & 60 & 61.9 & 51 & 85.0 & 0.002 \\
\hline \multirow{2}{*}{ Neoadjuvant regimen } & Other & 55 & 35.0 & 30 & 30.9 & 25 & 41.7 & \multirow{2}{*}{0.231} \\
\hline & FLOT & 102 & 65.0 & 67 & 69.1 & 35 & 58.3 & \\
\hline & Near-complete & 14 & 8.9 & 12 & 12.4 & 2 & 3.3 & \\
\hline Pathological response & Partial & 57 & 36.3 & 43 & 44.3 & 14 & 23.3 & 0.001 \\
\hline & Poor & 86 & 54.8 & 42 & 43.3 & 44 & 73.3 & \\
\hline $\begin{array}{l}\text { Neoadjuvant treatment } \\
\text { completion }\end{array}$ & Yes & 144 & 91.7 & 91 & 93.8 & 53 & 88.3 & 0.246 \\
\hline & Yes & 60 & 38.2 & 24 & 24.7 & 36 & 60.0 & $<0.001$ \\
\hline & Locoregional & 7 & 11.7 & 5 & 20.8 & 2 & 5.6 & 0.104 \\
\hline & Liver & 25 & 41.7 & 11 & 45.8 & 14 & 38.9 & 0.593 \\
\hline Recurrence & Peritoneum & 28 & 46.7 & 8 & 33.3 & 20 & 55.6 & 0.091 \\
\hline & Distant LN & 3 & 5.0 & 3 & 12.5 & 0 & 0.0 & 0.033 \\
\hline & Lung & 11 & 18.3 & 2 & 8.3 & 9 & 25.0 & 0.102 \\
\hline
\end{tabular}




\section{Cureus}

$\begin{array}{|lllllllll|} & \begin{array}{l}\text { Others (brain, bone, muscle, } \\ \text { surrenal) }\end{array} & 2 & 3.3 & 2 & 8.3 & 0 & 0.0 & 0.078 \\ & \text { Chemotherapy } & 45 & 75.0 & 18 & 75.0 & 27 & 75.0 & 1.000 \\ \text { First-line treatment } & \text { Best supportive care } & 15 & 25.0 & 6 & 25.0 & 9 & 25.0 & \\ & \text { Exitus } & 46 & 29.3 & 18 & 18.6 & 28 & 46.7 & <0.001 \\ \text { Status at the last follow-up } & \text { Alive } & 111 & 70.7 & 79 & 81.4 & 32 & 53.3 & \end{array}$

\section{TABLE 1: Patient characteristics}

ECOG PS: Eastern Cooperative Oncology Group Performance Status; CA 19-9: carbohydrate antigen 19-9; CEA: carcinoembryonic antigen; ypLNR: lymph node ratio; LN: lymph node; ypT: tumor stage; ypN: lymph node stage; SD: standard deviation

\begin{tabular}{|c|c|c|c|c|c|c|c|}
\hline \multirow{2}{*}{ Characteristics } & & \multicolumn{3}{|c|}{ Multivariate analysis for DFS } & \multicolumn{3}{|c|}{ Multivariate Analysis for OS } \\
\hline & & HR & $95 \% \mathrm{Cl}$ for $\mathrm{HR}$ & P-value & HR & $95 \% \mathrm{Cl}$ for HR & P-value \\
\hline Age & Years & & & & 1.033 & 0.996-1.071 & 0.075 \\
\hline \multirow{4}{*}{ Tumor localization } & $1 / 3$ (reference) & & & & 1.000 & & 0.053 \\
\hline & $2 / 3$ & & & & 1.421 & $0.614-3.288$ & 0.411 \\
\hline & $3 / 3$ & & & & 2.649 & $0.884-7.933$ & 0.082 \\
\hline & Linitis plastica & & & & 2.657 & $1.252-5.638$ & 0.011 \\
\hline Lymphovascular invasion & positive vs. negative & 2.567 & $1.023-6.435$ & 0.044 & 2.164 & $0.866-5.402$ & 0.098 \\
\hline \multirow{3}{*}{ Neoadjuvant response } & Poor response (reference) & 1.000 & & 0.024 & 1.000 & & 0.034 \\
\hline & Partial response & 0.429 & $0.055-3.338$ & 0.419 & 0.800 & 0.089-7.118 & 0.841 \\
\hline & Near-complete response & 0.345 & $0.158-0.753$ & 0.007 & 0.276 & $0.104-0.729$ & 0.009 \\
\hline Adjuvant treatment & Yes vs. no & 0.250 & $0.106-0.588$ & 0.001 & 0.272 & $0.114-0.645$ & 0.003 \\
\hline LNR & $>0.255$ vs. $\leq 0.255$ & 2.418 & $1.334-4.381$ & 0.004 & 2.268 & $1.166-4.407$ & 0.016 \\
\hline
\end{tabular}

TABLE 2: Multivariate analysis for survival

DFS: disease-free survival; OS: overall survival; HR: hazard ratio; Cl: confidence interval; LNR: lymph node ratio

\begin{tabular}{|c|c|c|c|c|c|c|c|}
\hline \multirow{2}{*}{ Characteristics } & & \multicolumn{3}{|c|}{ Univariate analysis for DFS } & \multicolumn{3}{|c|}{ Univariate analysis for OS } \\
\hline & & HR & $\begin{array}{l}95 \% \mathrm{Cl} \text { for } \\
\text { HR }\end{array}$ & $\begin{array}{l}\mathrm{P} \text { - } \\
\text { value }\end{array}$ & HR & $\begin{array}{l}95 \% \mathrm{Cl} \text { for } \\
\mathrm{HR}\end{array}$ & $\begin{array}{l}\mathrm{P} \text { - } \\
\text { value }\end{array}$ \\
\hline Gender & Men vs. women & 1.617 & $0.929-2.814$ & 0.089 & 1.807 & $0.931-3.504$ & 0.080 \\
\hline Age & Years & 1.018 & 0.989-1.049 & 0.222 & 1.023 & $0.986-1.061$ & 0.214 \\
\hline Smoking status & Yes vs. no & 0.904 & $0.501-1.631$ & 0.738 & 0.902 & $0.469-1.732$ & 0.756 \\
\hline ECOG PS & 1 vs. 0 & 1.148 & $0.519-2.538$ & 0.734 & 1.806 & $0.792-4.116$ & 0.159 \\
\hline Clinical stage & 2 vs. 3 & 0.638 & $0.198-2.045$ & 0.449 & 0.645 & 0.198-2.089 & 0.464 \\
\hline CEA & ng/mL & 1.010 & $1.001-1.019$ & 0.044 & 1.002 & $0.989-1.014$ & 0.767 \\
\hline CA $19-9$ & $\mathrm{U} / \mathrm{mL}$ & 1.000 & $0.999-1.001$ & 0.293 & 0.999 & $0.999-1.001$ & 0.735 \\
\hline Lauren classification & Diffuse vs. intestinal & 1.096 & $0.568-2.111$ & 0.785 & 0.858 & $0.382-1.922$ & 0.709 \\
\hline
\end{tabular}




\section{Cureus}

\begin{tabular}{|c|c|c|c|c|c|c|c|}
\hline \multirow{4}{*}{ Tumor localization } & 1/3 (reference) & \multicolumn{2}{|l|}{1.000} & 0.472 & \multicolumn{2}{|l|}{1.000} & 0.055 \\
\hline & $2 / 3$ & 0.924 & $0.469-1.820$ & 0.819 & 1.524 & $0.694-3.348$ & 0.294 \\
\hline & $3 / 3$ & 1.450 & $0.786-2.673$ & 0.234 & 2.327 & $1.115-4.855$ & 0.024 \\
\hline & Linitis plastica & 1.568 & $0.597-4.117$ & 0.361 & 3.339 & $1.187-9.390$ & 0.022 \\
\hline \multirow{3}{*}{ Histology } & $\begin{array}{l}\text { Tubular and papillary adenocarcinoma } \\
\text { (reference) }\end{array}$ & 1.000 & & 0.008 & 1.000 & & 0.007 \\
\hline & Mucinous carcinoma & 0.579 & $0.224-1.489$ & 0.256 & 0.456 & $0.136-1.521$ & 0.202 \\
\hline & Ring cell carcinoma & 2.039 & $1.183-3.513$ & 0.010 & 2.266 & $1.215-4.224$ & 0.010 \\
\hline \multirow{3}{*}{ Grade } & 1 & 1.000 & & 0.218 & 1.000 & & 0.121 \\
\hline & 2 & 0.553 & $0.165-1.856$ & 0.338 & 0.369 & $0.103-1.311$ & 0.123 \\
\hline & 3 & 1.289 & $0.393-4.218$ & 0.675 & 1.552 & $0.470-5.125$ & 0.470 \\
\hline Gastrectomy & Total vs. subtotal & 1.148 & $0.687-1.916$ & 0.598 & 0.976 & $0.543-1.753$ & 0.935 \\
\hline LN dissection & D2 vs. D1 & 0.558 & $0.327-0.951$ & 0.032 & 0.665 & $0.361-1.221$ & 0.188 \\
\hline Perineural invasion & Positive vs. negative & 2.661 & $1.347-5.255$ & 0.005 & 2.627 & $1.220-5.962$ & 0.013 \\
\hline $\begin{array}{l}\text { Lymphovascular } \\
\text { invasion }\end{array}$ & Positive vs. negative & 3.064 & $1.504-6.242$ & 0.002 & 3.049 & $1.352-6.871$ & 0.007 \\
\hline \multirow{3}{*}{ урTNM } & I (reference) & 1.000 & & 0.001 & 1.000 & & 0.029 \\
\hline & II & 0.375 & $0.068-2.069$ & 0.260 & 0.422 & $0.076-2.319$ & 0.321 \\
\hline & III & 3.321 & $1.564-9.553$ & 0.013 & 1.663 & $0.401-6.896$ & 0.483 \\
\hline урт & 3-4 vs. $0-2$ & 2.729 & $1.988-5.528$ & 0.033 & 1.855 & $0.664-5.175$ & 0.238 \\
\hline Tumor diameter & $\mathrm{mm}$ & 1.035 & $0.932-1.148$ & 0.520 & 1.019 & $0.902-1.150$ & 0.759 \\
\hline \multirow{3}{*}{ ypN } & 1 (reference) & 1.000 & & $<0.001$ & 1.000 & & 0.004 \\
\hline & 2 & 3.256 & $1.402-7.761$ & 0.006 & 2.256 & $0.886-5.737$ & 0.088 \\
\hline & 3 & 4.894 & $\begin{array}{l}2.257- \\
10.607\end{array}$ & $<0.001$ & 3.994 & $1.717-9.286$ & 0.001 \\
\hline $\begin{array}{l}\text { Number of LNs } \\
\text { removed }\end{array}$ & & 0.996 & $0.977-1.015$ & 0.679 & 0.998 & $0.976-1.019$ & 0.822 \\
\hline $\begin{array}{l}\text { Number of positive } \\
\text { LNs }\end{array}$ & & 1.055 & $1.026-1.084$ & $<0.001$ & 1.058 & $1.025-1.092$ & $<0.001$ \\
\hline \multirow{3}{*}{ ERBB2 } & 0-1 (reference) & 1.000 & & 0.935 & 1.000 & & 0.978 \\
\hline & 2 & 0.950 & $0.378-2.382$ & 0.912 & 0.894 & $0.318-2.509$ & 0.832 \\
\hline & 3 & 1.194 & $0.430-3.314$ & 0.734 & 0.984 & $0.302-3.203$ & 0.978 \\
\hline Neoadjuvant regimen & Other vs. FLOT & 1.097 & $0.640-1.877$ & 0.736 & 1.288 & $0.671-2.473$ & 0.446 \\
\hline \multirow{3}{*}{$\begin{array}{l}\text { Neoadjuvant } \\
\text { response }\end{array}$} & Poor response (reference) & 1.000 & & $<0.001$ & 1.000 & & 0.001 \\
\hline & Partial response & 0.157 & $0.022-1.139$ & 0.037 & 0.247 & $0.033-1.806$ & 0.168 \\
\hline & Near complete response & 0.236 & $0.113-0.481$ & $<0.001$ & 0.181 & $0.071-0.461$ & $<0.001$ \\
\hline Adjuvant treatment & Yes vs. no & 0.364 & $0.176-0.751$ & 0.006 & 0.401 & $0.176-0.908$ & 0.028 \\
\hline LNR & $>0.255$ vs. $\leq 0.255$ & 2.848 & $1.697-4.779$ & $<0.001$ & 2.816 & $1.548-5.121$ & 0.001 \\
\hline
\end{tabular}

\section{TABLE 3: Univariate analysis for survival}

DFS: disease-free survival; OS: overall survival; HR: hazard ratio; Cl: confidence interval; ECOG PS: Eastern Cooperative Oncology Group Performance Status; CA 19-9: carbohydrate antigen 19-9; CEA: carcinoembryonic antigen; LN: lymph node; ypT: tumor stage; ypN: Iymph node stage 


\section{Cureus}

Patients with locally advanced gastric adenocarcinoma

$$
n=653
$$

\begin{tabular}{|c|c|}
\hline $\begin{array}{l}\text { Lymph node negative } \\
\text { after neoadjuvant } \\
\text { chemotherapy } n=\mathbf{1 5 2}\end{array}$ & $\begin{array}{l}\text { Lack of neoadjuvant } \\
\text { chemotherapy } \\
\qquad \mathbf{n =} \mathbf{3 1 2}\end{array}$ \\
\hline
\end{tabular}

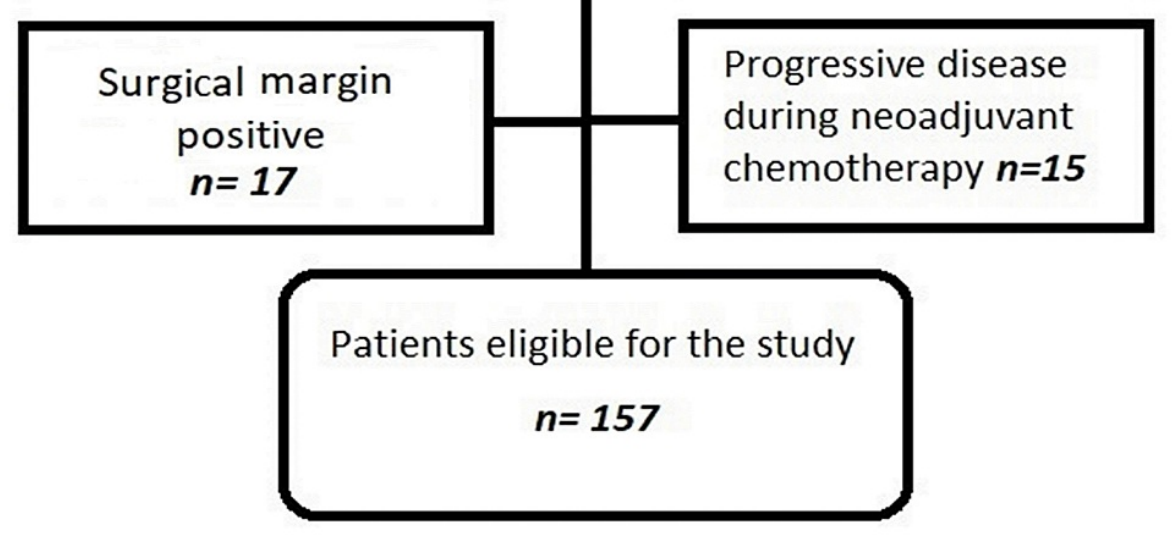

FIGURE 1: CONSORT flow diagram 


\section{Cureus}

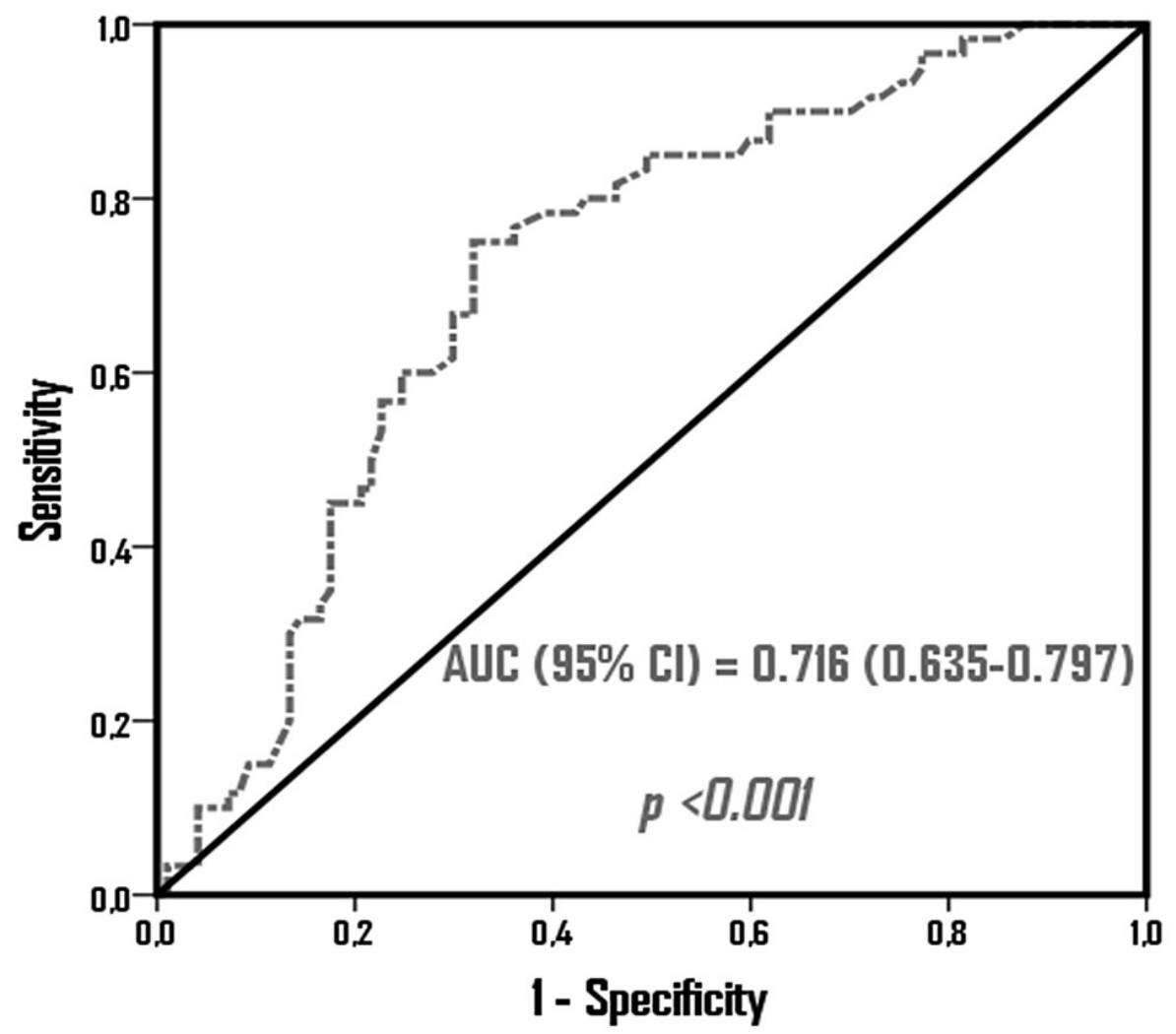

FIGURE 2: ROC curve analysis to verify the predictive power of ypLNR in predicting recurrence

ROC: receiver operating characteristic; AUC: area under the curve; ypLNR: lymph node ratio 


\section{Cureus}

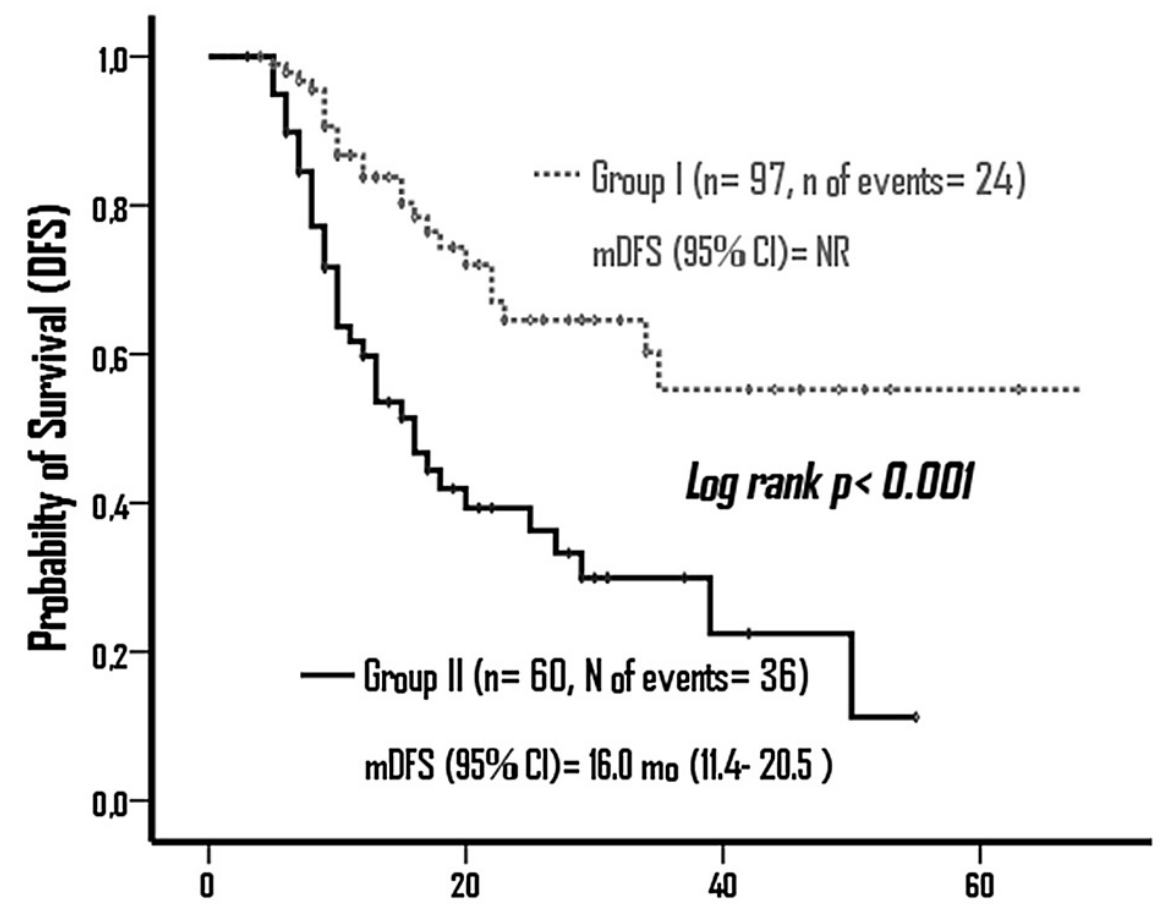

No. at risk

Time, Months

$\begin{array}{lllll}\text { Group I } & 97 & 31 & 11 & 4 \\ \text { Group II } & 60 & 15 & 2 & 0\end{array}$

FIGURE 3: Disease-free survival according to ypLNR groups ypLNR: lymph node ratio 


\section{Cureus}

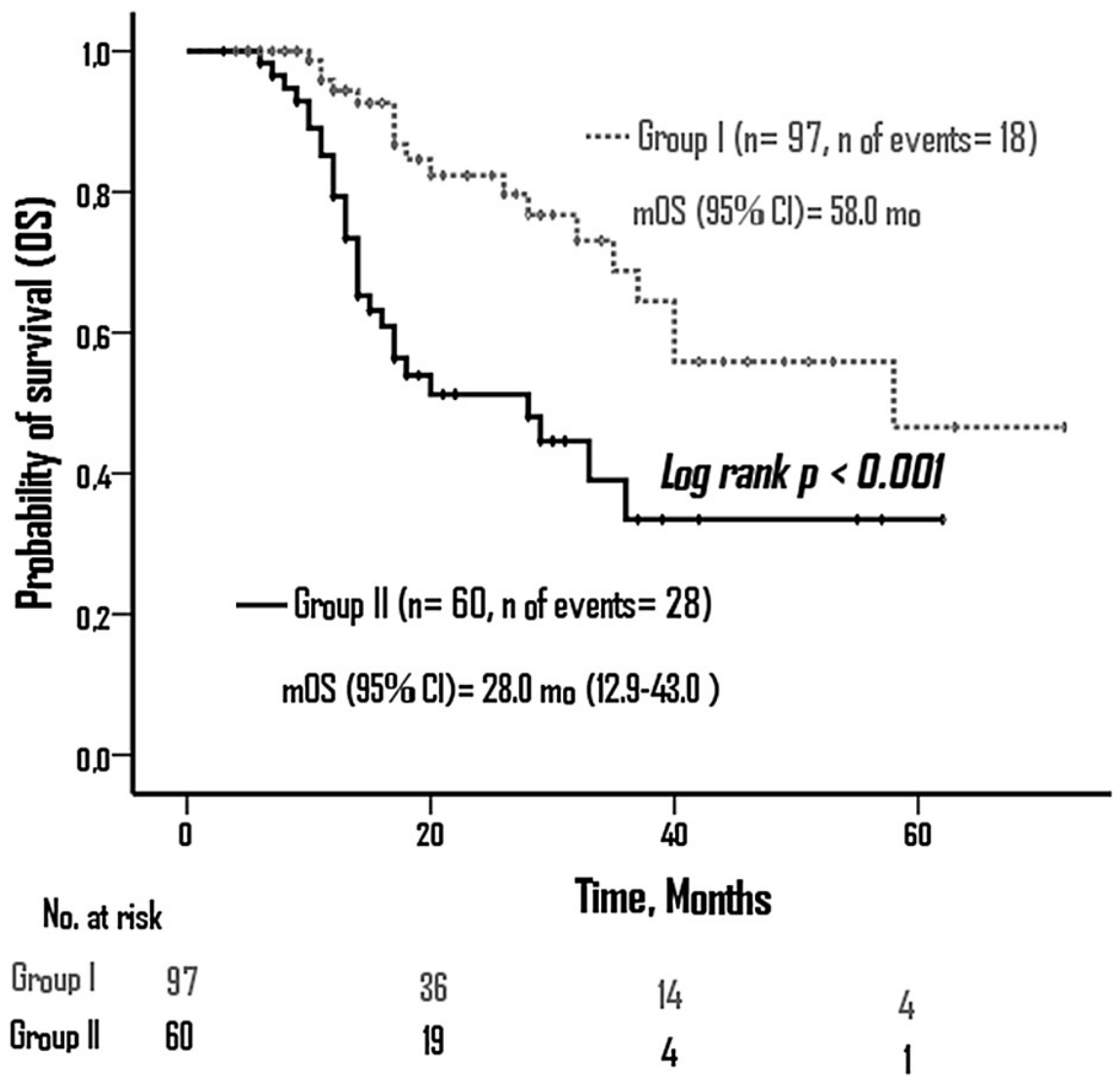

FIGURE 4: Overall survival according to ypLNR groups

ypLNR: lymph node ratio

\section{Discussion}

The present study investigated the effect of ypLNR on prognosis in patients with locally advanced GC who were operated on after NACT and were LN-positive postoperatively. The cut-off value for ypLNR was identified as 0.255 with $60 \%$ sensitivity and $76 \%$ specificity. Both DFS and OS were significantly better in patients with low ypLNR rates. Furthermore, ypLNR higher than 0.255 was observed to increase the risk of recurrence by 2.4 times and the risk of mortality by 2.26 times.

Previous studies have investigated the association between LNR and survival in several types of solid tumors [21-23]. In GC, the association between LNR and survival has been investigated mostly in patients not treated with neoadjuvant therapy [24]. Studies have demonstrated that LNR may be used as a more convenient and reliable parameter than TNM classification in operated patients with locally advanced GC. Moreover, LNR has been shown to be potentially prognostic for liver and peritoneal metastasis in this patient group [25-27].

In the literature, the only study conducted with patients treated with NACT appears to be a retrospective single-center study by Rawicz-Pruszyński et al. involving 95 patients. Their study looked at the effect of NACT on ypLNR in GC patients. The authors reported that tumor diameter of $>3.5 \mathrm{~cm}$, Lauren intestinal subtype, a lack of response to NACT, serosal infiltration, LN metastases, and distant metastases were significantly associated with higher ypLNR [18]. Eren et al. showed that higher ypLNR was associated with worse survival in their study in which patients received modified docetaxel, cisplatin, and fluorouracil (mDCF) preoperatively [28]. Higher ypLNR was associated with significantly shorter DFS and OS in our study. ypLNR was determined as a factor that affects survival both in the univariate analysis and multivariate analysis. There was no difference between ypLNR groups in terms of recurrence localization.

Studies have shown the independent effect of pT stage and tumor size on survival in patients operated on without receiving neoadjuvant treatment [29-31]. On the other hand, studies in patients receiving NACT revealed an effect on survival with ypN rather than the ypT stage [32,33]. In our study, the multivariate analysis did not show any effect of the ypT stage on survival. While the ypN stage appeared to affect survival in the univariate analysis, ypN was observed to lose its importance when added together with ypLNR in the 
multivariate analysis.

Conflicting study results have been reported concerning the effect of NACT response on survival. Most studies have shown increased survival with increased tumor response [32,34,35]. However, Smyth et al. did not find a correlation between tumor response and survival [16]. Our study, unlike other studies, included only LN-positive patients, and in line with most of the other studies, we observed increased survival with increased response to NACT. In addition, we observed significantly increased DFS and OS associated with NACT completion.

Patients with positive surgical margins were not included in the present study to avoid a potential effect on results. This study has some limitations, including the retrospective design and enrollment from only two centers. Also, our study population consisted only of Turkish patients.

Increased tumor response in GC patients operated on after NACT, NACT completion, and ypLNR rates under 0.255 were observed to significantly improve both DFS and OS in the present study. In conclusion, ypLNR has been identified as a more significant prognostic marker than the ypN stage in patients who remain LN-positive after NACT. We believe ypLNR may be used as a conveniently estimated prognostic marker in this group of patients. Our study results warrant confirmation via larger studies across different populations.

\section{Conclusions}

Based on our findings, ypLNR is a more significant prognostic marker than the ypN stage in patients who remain LN-positive after NACT. We believe ypLNR may be used as a conveniently estimated prognostic marker in this group of patients. Larger studies across different populations need to be conducted in order to confirm our findings.

\section{Additional Information}

\section{Disclosures}

Human subjects: Consent was obtained or waived by all participants in this study. Ethics Committee of the University of Health Sciences, Prof. Dr. Cemil Taşcıoğlu Istanbul City Training and Research Hospital issued approval 48670771-514.10. This study was approved by the Ethics Committee of the University of Health Sciences, Prof. Dr. Cemil Taşcıoğlu Istanbul City Training and Research Hospital. Animal subjects: All authors have confirmed that this study did not involve animal subjects or tissue. Conflicts of interest: In compliance with the ICMJE uniform disclosure form, all authors declare the following: Payment/services info: All authors have declared that no financial support was received from any organization for the submitted work. Financial relationships: All authors have declared that they have no financial relationships at present or within the previous three years with any organizations that might have an interest in the submitted work. Other relationships: All authors have declared that there are no other relationships or activities that could appear to have influenced the submitted work.

\section{References}

1. Wanebo HJ, Kennedy BJ, Chmiel J, Steele G Jr, Winchester D, Osteen R: Cancer of the stomach. A patient care study by the American College of Surgeons. Ann Surg. 1993, 218:583-92. 10.1097/00000658-19932185000002

2. Fuchs CS, Mayer RJ: Gastric carcinoma. N Engl J Med. 1995, 333:32-41. 10.1056/NEJM199507063330107

3. Bray F, Ferlay J, Soerjomataram I, Siegel RL, Torre LA, Jemal A: Global cancer statistics 2018: GLOBOCAN estimates of incidence and mortality worldwide for 36 cancers in 185 countries. CA Cancer J Clin. 2018, 68:394-42. 10.3322/caac.21492

4. Earle CC, Maroun JA: Adjuvant chemotherapy after curative resection for gastric cancer in non-Asian patients: revisiting a meta-analysis of randomised trials. Eur J Cancer. 1999, 35:1059-64. 10.1016/s09598049(99)00076-3

5. Cunningham D, Allum WH, Stenning SP, et al.: Perioperative chemotherapy versus surgery alone for resectable gastroesophageal cancer. N Engl J Med. 2006, 355:11-20. 10.1056/NEJMoa055531

6. Al-Batran SE, Hofheinz RD, Pauligk C, et al.: Histopathological regression after neoadjuvant docetaxel, oxaliplatin, fluorouracil, and leucovorin versus epirubicin, cisplatin, and fluorouracil or capecitabine in patients with resectable gastric or gastro-oesophageal junction adenocarcinoma (FLOT4-AIO): results from the phase 2 part of a multicentre, open-label, randomised phase 2/3 trial. Lancet Oncol. 2016, 17:1697-708. 10.1016/S1470-2045(16)30531-9

7. Ajani JA, Mansfield PF, Janjan N, et al.: Multi-institutional trial of preoperative chemoradiotherapy in patients with potentially resectable gastric carcinoma. J Clin Oncol. 2004, 22:2774-80. 10.1200/JCO.2004.01.015

8. Ronellenfitsch U, Schwarzbach M, Hofheinz R, Kienle P, Kieser M, Slanger TE, Jensen K: Perioperative chemo(radio)therapy versus primary surgery for resectable adenocarcinoma of the stomach, gastroesophageal junction, and lower esophagus. Cochrane Database Syst Rev. 2013, 5:CD008107. 10.1002/14651858.CD008107.pub2

9. Nio Y, Tsubono M, Kawabata K, et al.: Comparison of survival curves of gastric cancer patients after surgery according to the UICC stage classification and the General Rules for Gastric Cancer Study by the Japanese Research Society for gastric cancer. Ann Surg. 1993, 218:47-53. 10.1097/00000658-199307000-00008 
10. Sano T, Coit DG, Kim HH, et al.: Proposal of a new stage grouping of gastric cancer for TNM classification: International Gastric Cancer Association staging project. Gastric Cancer. 2017, 20:217-25. 10.1007/s10120016-0601-9

11. Spolverato G, Ejaz A, Kim Y, et al.: Prognostic performance of different lymph node staging systems after curative intent resection for gastric adenocarcinoma. Ann Surg. 2015, 262:991-8. 10.1097/SLA.0000000000001040

12. Kajitani T: The general rules for the gastric cancer study in surgery and pathology. Part I. Clinical classification. Jpn J Surg. 1981, 11:127-39. 10.1007/BF02468883

13. Cuschieri A, Fayers P, Fielding J, Craven J, Bancewicz J, Joypaul V, Cook P: Postoperative morbidity and mortality after D1 and D2 resections for gastric cancer: preliminary results of the MRC randomised controlled surgical trial. The Surgical Cooperative Group. Lancet. 1996, 347:995-9. 10.1016/s01406736(96)90144-0

14. Smith DD, Schwarz RR, Schwarz RE: Impact of total lymph node count on staging and survival after gastrectomy for gastric cancer: data from a large US-population database. J Clin Oncol. 2005, 23:7114-24. 10.1200/JCO.2005.14.621

15. Seevaratnam R, Bocicariu A, Cardoso R, et al.: How many lymph nodes should be assessed in patients with gastric cancer? A systematic review. Gastric Cancer. 2012, 15:S70-88. 10.1007/s10120-012-0169-y

16. Smyth EC, Fassan M, Cunningham D, et al.: Effect of pathologic tumor response and nodal status on survival in the Medical Research Council Adjuvant Gastric Infusional Chemotherapy Trial. J Clin Oncol. 2016, 34:2721-7. 10.1200/JCO.2015.65.7692

17. Sawayama H, Iwatsuki M, Kuroda D, et al.: The association of the lymph node ratio and serum carbohydrate antigen 19-9 with early recurrence after curative gastrectomy for gastric cancer. Surg Today. 2018, 48:9941003. 10.1007/s00595-018-1684-1

18. Rawicz-Pruszyński K, Ciseł B, Mlak R, et al.: The role of the lymph node ratio in advanced gastric cancer after neoadjuvant chemotherapy. Cancers (Basel). 2019, 11:1914. 10.3390/cancers11121914

19. Alatengbaolide, Lin D, Li Y, et al.: Lymph node ratio is an independent prognostic factor in gastric cancer after curative resection (R0) regardless of the examined number of lymph nodes. Am J Clin Oncol. 2013, 36:325-30. 10.1097/COC.0b013e318246b4e9

20. Kutlu OC, Watchell M, Dissanaike S: Metastatic lymph node ratio successfully predicts prognosis in western gastric cancer patients. Surg Oncol. 2015, 24:84-8. 10.1016/j.suronc.2015.03.001

21. Sakin A, Aldemir MN: Lymph node ratio predicts long-term survival in lymph node-positive breast cancer . Eur J Breast Health. 2020, 16:270-5. 10.5152/ejbh.2020.5809

22. Xiao W, Liang $\mathrm{H}$, Zhang $\mathrm{H}$, et al.: Ratio between negative and positive lymph nodes is a novel prognostic indicator for patients with esophageal cancer: A Surveillance, Epidemiology and End Results database analysis. Thorac Cancer. 2020, 11:3490-50. 10.1111/1759-7714.13688

23. Lyu S, Li L, Zhao X, Ren Z, Cao D, He Q: Prognostic impact of lymph node parameters in distal cholangiocarcinoma after pancreaticoduodenectomy. World J Surg Oncol. 2020, 18:262. 10.1186/s12957020-02040-1

24. Zhu J, Xue Z, Zhang S, et al.: Integrated analysis of the prognostic role of the lymph node ratio in nodepositive gastric cancer: a meta-analysis. Int J Surg. 2018, 57:76-83. 10.1016/j.ijsu.2018.08.002

25. Zhou Y, Zhang J, Cao S, Li Y: The evaluation of metastatic lymph node ratio staging system in gastric cancer. Gastric Cancer. 2013, 16:309-17. 10.1007/s10120-012-0190-1

26. Marchet A, Mocellin S, Ambrosi A, et al.: The ratio between metastatic and examined lymph nodes (N ratio) is an independent prognostic factor in gastric cancer regardless of the type of lymphadenectomy: results from an Italian multicentric study in 1853 patients. Ann Surg. 2007, 245:543-52. 10.1097/01.sla.0000250423.43436.e1

27. Li MX, Jin ZX, Zhou JG, et al.: Prognostic value of lymph node ratio in patients receiving combined surgical resection for gastric cancer liver metastasis: results from two national centers in China. Medicine (Baltimore). 2016, 95:e3395. 10.1097/MD.0000000000003395

28. Eren T, Karaçin C, Uçar G, et al.: Efficacy of the combination of modified docetaxel, cisplatin and fluorouracil in locally advanced gastric cancer: evaluation of real-life outcomes. UHOD. 2020, 30:23-31. 10.4999/uhod.203959

29. Yang Y, Ma Y, Xiang X, Xing P, Wu Y, Zhang L, Tian Y: The prognostic value of the lymph node ratio for local advanced gastric cancer patients with intensity-modulated radiation therapy and concurrent chemotherapy after radical gastrectomy in China. Radiat Oncol. 2020, 15:237. 10.1186/s13014-020-01687-0

30. Aurello P, D'Angelo F, Rossi S, et al.: Classification of lymph node metastases from gastric cancer: comparison between $\mathrm{N}$-site and $\mathrm{N}$-number systems. Our experience and review of the literature. Am Surg. 2007, 73:359-66.

31. Shiraishi N, Sato K, Yasuda K, Inomata M, Kitano S: Multivariate prognostic study on large gastric cancer. J Surg Oncol. 2007, 96:14-8. 10.1002/jso.20631

32. Stark AP, Ikoma N, Chiang YJ, et al.: Characteristics and survival of gastric cancer patients with pathologic complete response to preoperative therapy. Ann Surg Oncol. 2019, 26:3602-10. 10.1245/s10434-019-076388

33. Ikoma N, Hofstetter WL, Estrella JS, et al.: The ypT category does not impact overall survival in node negative gastric cancer. J Surg Oncol. 2018, 117:1721-8. 10.1002/jso.25081

34. Peng L, Yang W, Zhang Z, Zhi C, Zhou X, Liu H, Hua Y: Prognostic analysis of neoadjuvant chemotherapy for locally advanced gastric cancer with propensity score matching method (Article in Chinese). Zhonghua Wei Chang Wai Ke Za Zhi. 2018, 21:1148-53.

35. Coimbra FJF, de Jesus VHF, Ribeiro HSC, et al.: Impact of ypT, ypN, and adjuvant therapy on survival in gastric cancer patients treated with perioperative chemotherapy and radical surgery. Ann Surg Oncol. 2019, 26:3618-26. 10.1245/s10434-019-07454-0 